\title{
A combination of long term fragmentation and glacial persistence drove the evolutionary history of the Italian wall lizard Podarcis siculus
}

\author{
${\text { Gabriele Senczuk }{ }^{1 *} \text { (D), Paolo Colangelo }}^{1,2}$, Emanuela De Simone ${ }^{1}$, Gaetano Aloise ${ }^{3}$ and Riccardo Castiglia ${ }^{1}$
}

\begin{abstract}
Background: The current distribution of genetic diversity is the result of a vast array of microevolutionary processes, including short-term demographic and ecological mechanisms and long-term allopatric isolation in response to Quaternary climatic fluctuations. We investigated past processes that drove the population differentiation and spatial genetic distribution of the Italian wall lizard Podarcis siculus by means of sequences of mitochondrial cytb $(n=277$ from 115 localities) and nuclear mc1r and $\beta$-fibint7genes ( $n=262$ and $n=91$, respectively) from all its distribution range. The pattern emerging from the genetic data was compared with current and past (last glacial maximum) species distribution modeling (SDM).

Results: We identified seven deeply divergent parapatric clades which presumably remained isolated in different refugia scattered mainly throughout the Tyrrhenian coast. Conversely, the Adriatic coast showed only two haplogroups with low genetic variability. These results appear to agree with the SDM prediction at the last glacial maximum (LGM) indicating a narrow area of habitat suitability along the Tyrrhenian coast and much lower suitability along the Adriatic one. However, the considerable land exposure of the Adriatic coastline favored a glacial colonization of the Balkan Peninsula.

Conclusions: Our population-level historical demography showed a common trend consistent with glacial expansions and regional persistence during the last glacial maximum. This complex genetic signature appears to be inconsistent with the expectation of the expansion-contraction model and post-LGM (re)colonizations from southern refugia. Hence it is one of an increasing number of cases in which these assumptions are not met, indicating that long-term fragmentation and pre-LGM events such as glacial persistence were more prominent in shaping genetic variation in this temperate species.
\end{abstract}

Keywords: Italian wall lizard, Phylogeography, Glacial expansion, Podarcis siculus, Niche modelling, Evolutionary history

\section{Background}

The cyclic population expansions and contractions primed by the Quaternary climatic oscillation are considered one of the most important processes that led to the current distribution of genetic variation across different geographic areas [1-5]. However, the huge amount of molecular data made available in recent years has led to a vast

\footnotetext{
*Correspondence: gabriele.senczuk@uniroma1.it

"Dipartimento di Biologia e Biotecnologie "Charles Darwin", Università di

Roma LA SAPIENZA, sede di Anatomia comparata, Rome, Italy

Full list of author information is available at the end of the article
}

array of reconsiderations of microevolutionary processes that underlie the Pleistocene biogeography. Species responses to past climatic changes have been shown to be more variable than formerly thought, depending on species-specific physiological and ecological requirements [6-10]. Moreover, the climatic fluctuations acting in different and restricted topographic contexts generated a variety of contrasting evolutionary histories even at the population level $[11,12]$.

In the Mediterranean region, evidence from species inhabiting temperate and coastal areas suggested a certain 
flexibility of the expansion-contraction (EC) model of Pleistocene biogeography [1] and of the expectation of post-last glacial maximum (LGM) demographic expansions from southern refugia. These new perspectives suggest that some temperate species could have undergone attenuated or even reverse responses to glacial population contractions, with the absence of a postLGM (re)colonization pattern [13, 14]. This scenario appears plausible considering the strong sea level decrease (as much as 120-135 m) during the LGM, leading to the increase of Mediterranean coastal plains and favoring the formation of new suitable habitats with consequent population expansions.

Moreover, climate-driven cycles of allopatric differentiation within sub-refugia have now become the rule rather than the exception in molding patterns of genetic diversity in many Mediterranean species. Meanwhile, the perception of the maintenance of genetic diversity within a single macrorefugium, induced by population demographic stability, has gradually diminished in favor of the alternative 'refugia within refugia' scenario [15]. In fact, a growing number of taxa reveal evidence of strong phylogeographic structure within the main southern regions ([16-18] in the Iberian Peninsula; $[19,20]$ in the Italian Peninsula; $[11,21,22]$ in the Balkan Peninsula).

The Italian Peninsula is considered an important refugial area for many Mediterranean species due to its complex topography and wide latitudinal range [7, 23]. It has been repeatedly used as a study area to gain new insights into alternative microevolutionary processes generating patterns of genetic diversity [24]. Most of these studies confirmed a southern richness pattern of genetic diversity. In particular, the southernmost portion of the Italian Peninsula, the "Calabrian arc", has been called a melting pot of intraspecific diversity [25]. From the Pliocene to the Middle Pleistocene, especially during high-sea level interglacials, this area consisted of different islands corresponding to the Aspromonte, Sila and Pollino massifs $[26,27]$. The rise of highly divergent genetic lineages in these areas is probably closely linked to these paleo-island systems. However, the northern and central Apennines have also played a role in generating long-term genetic discontinuity, suggesting the presence of additional cryptic refugia in the central and northern portions of the Italian Peninsula [28, 29].

On the whole, the genetic population structure of many species inhabiting the Italian Peninsula suggests a vast assortment of long-term allopatric fragmentations and recent short-term distributional and demographic rearrangements in response to environmental variability.

In the present study, we used the Italian wall lizard Podarcis siculus to investigate a part of this microevolutionary process by means of mtDNA and nuDNA sequences in a phylogeographic framework. The Italian wall lizard is a good model to elucidate the historical events shaping the genetic architecture in the Italian Peninsula. It is widespread and very abundant in peninsular Italy, on the large islands Sicily, Sardinia and Corsica, and along the northern part of the eastern Adriatic coast and many Adriatic islands. Podnar et al. [30] recognized six main clades on the basis of mitochondrial data: three in southern Italy (Sicily and Calabria) and three in centralnorthern Italy and Croatia. Although their study included many Adriatic regions of Croatia and some southern Italian populations, much of the Italian peninsular area was not investigated.

Therefore, the aim of our study was to determine the long-term or recent microevolutionary processes most important in shaping the current genetic architecture across the entire refugial area. In doing so we wished to (i) assess the different demographic trends, distinguishing long-term isolation and/or allopatric differentiation within sub-refugia from recent expansion events, and (ii) shed more light on the importance of the Pleistocene environmental changes and consequent microevolutionary processes in structuring biodiversity in the Italian Peninsula.

\section{Methods}

\section{Sampling, laboratory procedures and molecular data}

The sampling took place from March 2013 to June 2015, during which we collected 277 samples from 115 localities covering most of the distribution area of Podarcis siculus in the Italian Peninsula and Sicily. The tissues were obtained by inducing autotomy after light pressure and were then stored in 96\% pure ethanol. All lizards were released at the capture location. The sampling was planned to complement already available data in order to achieve a better geographic coverage of the species distribution [30]. The geographic coordinates were recorded and high-resolution photos were taken for each individual. The geographic references and sample size of all sampled populations are given in Additional file 1: Table S1 and shown in Fig. 1.

Genomic DNA was extracted from all the tissue samples by means of the universal extraction protocol of Salah et al. [31] with incubation at $56{ }^{\circ} \mathrm{C}$ with proteinase $\mathrm{K}$ and DNA precipitation with isopropanol. The total genome was visually checked in electrophoretic runs. We used standard PCR protocols to amplify the mitochondrial fragment of cytochrome b (cytb) and two nuclear fragments, the Melanocortin receptor $1(\mathrm{mclr})$ and the $\beta$-fibrinogen intron 7 ( $\beta$-fibint7). The primers used for the amplifications and their respective references are reported in Table 1. As the presence of a pseudogene for $c y t b$ has been reported in this species [32], we modified the primers to be strongly specific to amplify only the mitochondrial $c y t b$ sequences. 


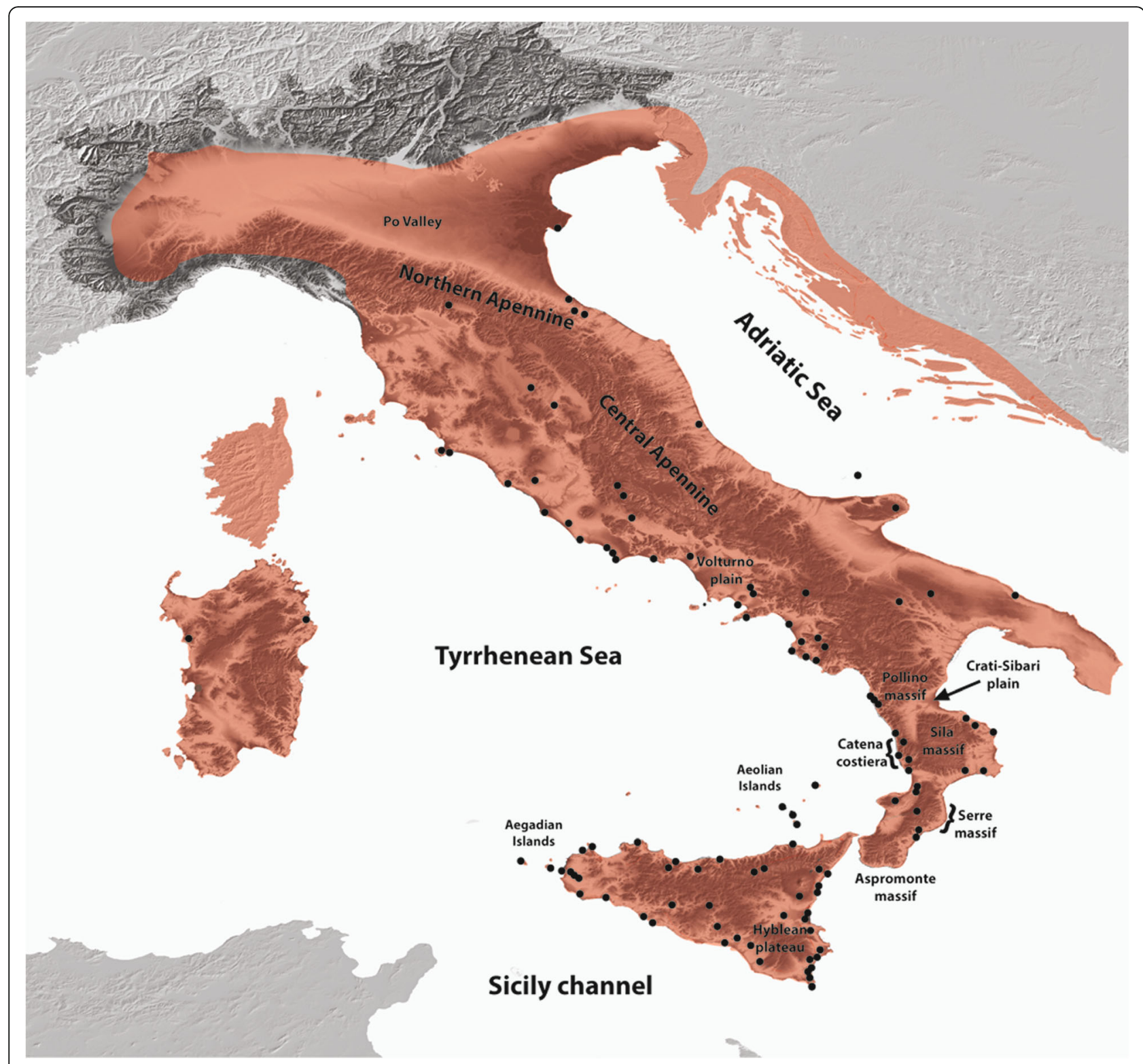

Fig. 1 Map of the study area showing the principal geographic features mentioned in the main text. The area in red corresponds to the geographic distribution of Podarcis siculus while the 115 new sampled locations are shown in black dots

Table 1 Pairs of primers used in this study with relative references

\begin{tabular}{|c|c|c|c|}
\hline Gene & Primer & Primer sequence & Reference \\
\hline \multirow[t]{2}{*}{ cytb } & CytF & $5^{\prime}$ - TTTGGATCACTATTRGGCCTCTGCC - 5' & \multirow[t]{2}{*}{ modified in this study from [30] } \\
\hline & H15425 & 5'- GGTITACAAGACCAGTGCTIT-3' & \\
\hline \multirow[t]{2}{*}{ me1r } & MC1R-PF & 5'- GGCNGCCATYGTCAANAACCGGAACC - 3' & \multirow[t]{2}{*}{ [103] } \\
\hline & MC1R-PR & 5'- CTCCGRAAGGCRTAAATNATGGGGTCCAC - 3' & \\
\hline \multirow[t]{2}{*}{$\beta$-fibint7 } & BFXF & $5^{\prime}$ - CAGYACTTTYGAYAGAGACAAYGATGG - 3' & [104] \\
\hline & FIB B17L & 5' - TCCCCAGTAGTATCTGCCATTAGGGTT - 3' & [105] \\
\hline
\end{tabular}


The resulting PCR products were purified with a Sure Clean (Bioline) purification kit. Sequencing reactions were run under Big-Dye TM Terminator cycling conditions by the commercial company Macrogen (www.macrogen. com). The electropherograms were then checked using FinchTV and minor changes were made by eye. IUPAC ambiguity codes were used to represent polymorphism of heterozygote individuals in nuclear sequences. For the nuclear sequences, we solved the gametic phases using the coalescent-based Bayesian algorithm in PHASE v.2.1 [33, 34] as implemented in DnaSPv.5.1 [35]. Since the $\beta$-fibint 7 showed the presence of INDELs polymorphisms, we first used the method described by Flot et al. [36] to determine the phase for sequences that were heterozygous for insertion or deletions (12 individuals). We then implemented the known phases in the coalescent-based Bayesian reconstruction. Three independent runs were conducted with burn-in at 1000 and 10,000 iterations, and thinning at each 100 steps. Only sequences with posterior probability $>0.75$ were included in the analysis. A final consensus alignment was computed for each marker with MEGA 5.0 [37]. When the final alignments were obtained, the number of haplotypes $(\mathrm{H})$, nucleotides $(\pi)$ and haplotype $(\mathrm{h})$ diversity were estimated using DnaSPv.5.1 for the overall mtDNA and nuDNA datasets and for each mtDNA clade revealed by the phylogenetic analysis. Since the statistical power of tests for recombination is generally low, two different methods were used to assess for nuclear recombination. The four-gamete test was performed to estimate the minimum number of recombination events obtaining confidence intervals at $95 \%$ by the coalescent algorithm implemented in DnaSP v.5.1.

Moreover, we also test the occurrence of recombination events through the Pairwise Homoplasy Index (phi) test implemented in the program splitstree v. 4 [38].

\section{Phylogeographic structureand time of divergence}

The phylogenetic analyses were carried out using a data set including $c y t b$ sequences generated in this study $(n=277$ specimens) and those available in GenBank ( $n=76$ specimens) from Podnar et al. [30], for a total alignment of 353 sequences. For the nuclear markers, here generated, we used a total of 524 sequences for $m c 1 r$ and 182 sequences for the $\beta$-fibint7 (accession numbers for each gene are reported in Additional file 2: Table $\mathrm{S} 2$ ).

To evaluate the best fitting substitution model for $c y t b$, we used jModeltestv.2 [39] considering the Akaike information criteria (AIC) and the Bayesian information criterion (BIC) as the most reliable criteria. The AIC and BIC criteria were concordant in assigning the general time reversible $(\mathrm{GTR}+\mathrm{I}+\mathrm{G})$ model to $c y t b$ as the best model of sequence evolution. BEAST v.1.8 [40] and MrBayes 3.2.6 [41] were used to generate a consensus tree based on coalescent Bayesian inference. BEAST was also used to obtain an estimate of the time to the most recent common ancestors (TMRCA). The choice of the right model could be challenging for such a data set; indeed the intraspecific framework could fall within a coalescent tree process which is more appropriate for a population-level analysis. However, the high levels of geographic structure and divergence between groups suggested that a Yule model would best fit the data. To infer the TMRCA, we assumed a relaxed molecular clock applying an uncorrelated lognormal distribution. Lacking a reliable calibration date on the root of the tree due to the absence of a fossil record, we applied a normal distribution to the mean-rate prior of the $c y t b$ mutation rate $(\mu=0.0175)$. Since this substitution rate was the average of substitution rates found in Podarcis lizards for the gene $c y t b$, the standard deviation of the normal prior distribution $(\mathrm{SD}=0.0014)$ was set in order to encompass the minimum value of 0.0145 [42] and the maximum value of 0.02 [43] proposed for the genus Podarcis. We run three independent MCMCs of 100 million generation sampling every 10.000 steps and the program TRACER v.1.6 [44] was used to check for the convergence of the parameters. The Bayesian analysis performed using MrBayes was run using four heated, one million step chains with an initial burn-in of 100,000 steps. Both Bayesian analyses were performed using Podarcis muralis as outgroup (accession number HQ652936).

Statistical parsimony networks were constructed under 95\% probability connection limits on each mtDNA clade identified by the phylogeny using TCS v.1.21 [45]. We used the same software to build additional further networks using the two nuDNA data sets.

Subsequently, we used a spatial principal component analysis (sPCA) as implemented in the R package ADEGENET v.1.2.8 [46] to explore the mtDNA spatial autocorrelation. The sPCA was applied to the whole data set and to each clade (see results for definition of the clades) to better highlight both the total and intraclade spatial distribution of the mtDNA. Unlike usual spatial analyses, eigenvalues of sPCA allow us to measure both the $c y t b$ genetic diversity (variance) and the spatial structure (spatial autocorrelation measured by Moran's I). This decomposition can also be used to choose which principal component can be employed to test, based on Monte Carlo permutations $(N=9999)$, the presence of global and/or local structure [46]. All the 346 coordinates were jittered (randomly shifted) by a factor of 10 (about $=100 \mathrm{~m}$ ) to avoid zero geographic distances between individuals from the same population. We used a Gabriel graph to generate a proximity connection network between populations. The sPCA variance was then plotted against the spatial autocorrelation (Moran's I) to visualize the existence of global 
and local structure. Global scores can identify genetically distinguishable clusters, clines in allele frequencies and intermediate samples, while local scores can detect local differentiation between neighboring sites.

Finally, to explore the genetic structure of the nuclear markers, we used SPADS v.1.0 [47] to perform the analysis of molecular variance (AMOVA), testing the level of nuclear genetic diversity among groups, among populations and within populations grouped according to the mtDNA clades revealed by the phylogenetic analysis.

\section{Historical demography and spatial diffusion}

Changes in population size over time were estimated by means of the multilocus Extended Bayesian Skyline Plot (EBSP) as implemented in BEAST v.1.8 [40]. The EBSP allows to combine information from different unlinked loci and thus to assess the uncertainty in the stochastic process (the coalescent), leading to improvement in the reliability of demographic inferences and a substantial reduction in estimating errors [48].

The EBSPs were performed maintaining the same prior settings used in the TMRCA analysis for the $c y t b$ (lognormal distribution; $\mu=0.0175, \mathrm{SD}=0.0014$ ), allowing the estimation of the nuclear genes.

The analysis was performed under a strict clock model, with unlinked substitution, clock and tree models for all the markers. We carried out three independent runs of $3 \times 10^{8}$ generations sampled every $30^{4}$ intervals for each clade with a sample size $>15$, using either the multilocus (with or without the $\beta$-fibint7) or only the mtDNA data set. Since the multilocus analysis with the $\beta$-fibint7 showed wider $95 \%$ highest posterior densities and large uninformative coalescent intervals due to the small sample size of this data set, we decided to eliminate this marker from the final runs. Conversely, the multilocus and mitochondrial EBSP yielded very similar results and we report only those from the multilocus analysis including the $m c 1 r$. The results were checked for diagnostic and parameters convergence using TRACER v.1.6 [44].

Finally, we used DnaSPv.5.1 to estimate Tajima's $D$ and Fu's $F s$ statistics and to assess their significance through 10,000 coalescent simulations under the hypothesis of population equilibrium and selective neutrality.

To reconstruct the location of ancestral areas and spatial diffusion from these, we used a continuous Relaxed Random Walk model (RRW) in a Bayesian phylogeographic analysis (BPA) as implemented in BEAST v. 1.8 (Drummond et al. 2012). We performed separated analysis on each clade (with a sample size $>15$ ) using the mtDNA data set applying GMRF Bayesian skyride prior [49] and a Cauchy RRW model of diffusion across branches [50]. MCMC chains were run for $3 \times 10^{8}$ generations sampling every $30^{4}$. Convergence of parameters for each run was assessed in TRACER v.1.6. The maximum clade credibility (MCC) was computed with TreeAnnotator v. 1.8. and was then used to visualize the spatial diffusion over time using the Time Slicer option in SPREAD v. 1.0.6 [51]. The 80\% highest posterior densities (HPD) were estimated and plotted at three specific time intervals (2.3 Mya, 1.3 Mya and 0.1 Mya).

\section{Species distribution modelling}

To better understand current and past habitat suitability of the species, we produced distribution models under different paleoclimatic conditions using species distribution models (SDM). The present-day occurrences of $P$. siculus were partially derived from the online database "Global Biodiversity Information System" (GBIF) and from the literature, but also from our georeferenced samples. The complete data set was trimmed to the spatial resolution of the environmental layers ( 2.5 arc min, about $4 \mathrm{~km})$ to avoid duplications within the same cell.

To infer habitat suitability for the species, we evaluated the 19 climatic variables available from the Worldclim database [52]. The number of environmental layers was chosen on the basis of our knowledge of the ecology and habitat preferences of the species. Hence the following six variables were selected: mean diurnal range (BIO2), temperature seasonality (BIO4), minimum temperature of coldest month (BIO6), temperature annual range (BIO7), annual precipitation (BIO12) and precipitation seasonality (BIO15). Pearson correlation coefficients were then calculated to assess the level of autocorrelation among the selected variables. The level of autocorrelation was fairly low, with no pairwise comparison with $r$ higher than 0.75 .

The past distribution of the Italian wall lizard was generated using ensemble forecasting as implemented in the R package 'biomod2' [53]. We used four different algorithms which estimate species distributions using environmental predictors together with species occurrences: Maxent [54, 55], Gradient Boosting Machines (GBM) [56], Generalized Linear Model (GLM) [57] and General Additive Model (GAM) [58]. The four algorithms were used to predict the species occurrence for both the present-day and the last glacial maximum (LGM, 26-20 Kya). We randomly split the records into two subsets including $70 \%$ of records to calibrate the models and $30 \%$ for evaluation [53]. This procedure was repeated 10 times and for each replicate a random selection of 10,000 background points was performed including the entire extent of occurrence of $P$. siculus. The predictivity of each model was assessed with AUC and TSS criteria. We performed model averaging by weighting the individual model projections by their AUC or TSS scores, retaining only those models with AUC $>0.7$ and TSS $>0.4$ to avoid poorly calibrated models. Projection of the model to the LGM condition 


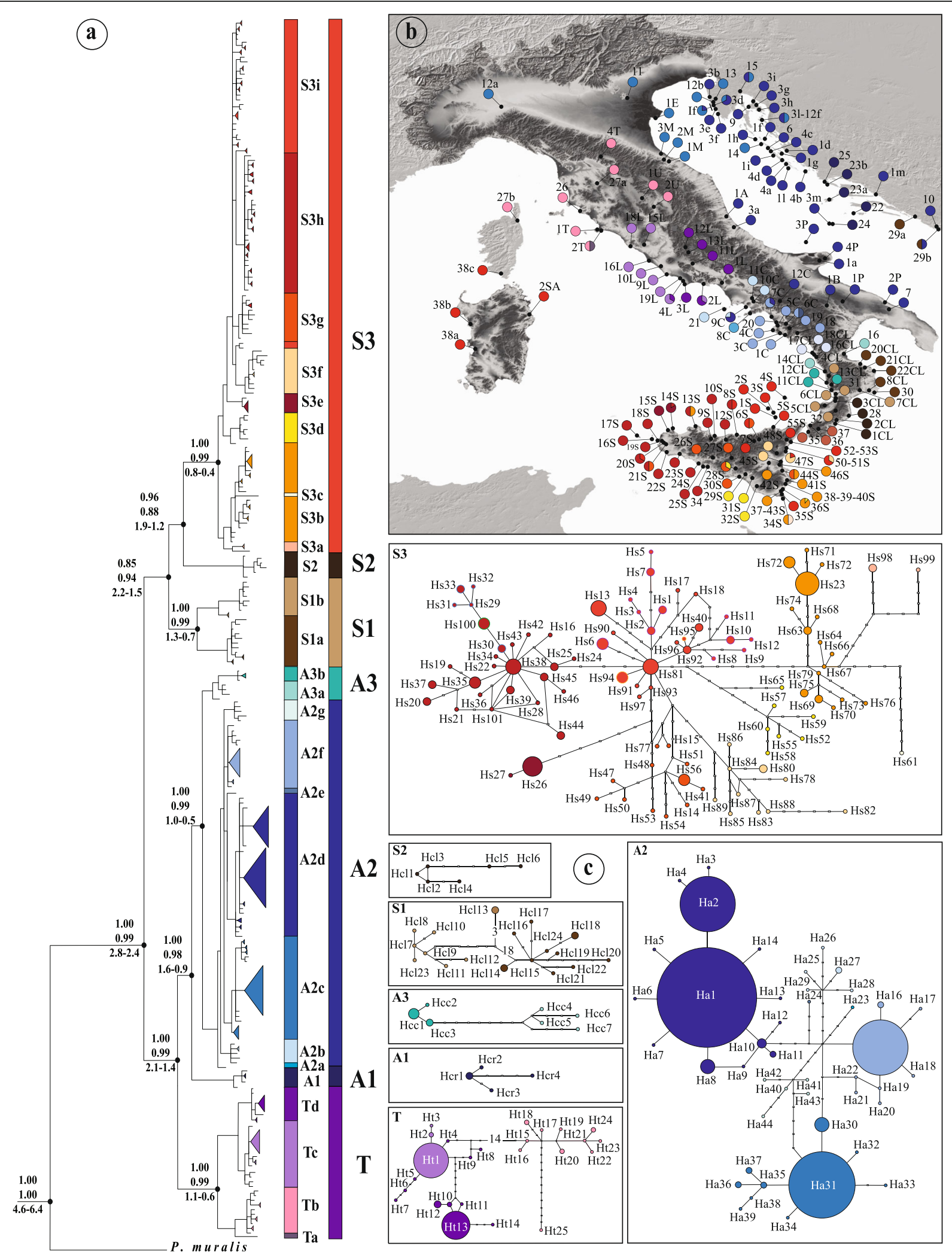

Fig. 2 (See legend on next page.) 
(See figure on previous page.)

Fig. 2 a Phylogenetic haplotype recostruction of the cytb data set obtained with MrBayes. At each node, MrBayes and BEAST posterior probability and TMRCA with 95\% highest posterior density (HPD) intervals are respectively reported. The seven clade such as its nested haplogroups are depicted with different colors. $\mathbf{b}$ Geographic distribution of the mtDNA haplotypes in Podarcis siculus. Pie diagrams shows the haplotype frequencies at each sampled location and are colored according to the main haplogroups identified by the phylogeny. c Statistical parsimony networks of the seven mtDNA clades identified by the phylogeny. Each circle size is proportional to their frequencies and each filled rectangles representing one substitution. The different colors within each network depict the principal identified haplogroups. Circum-Sicilian and Corsica-Sardinia haplotypes are reported in bold with different colors (violet: Aeolian haplotypes; blue: Aegadian haplotypes, green: Sardinia and Corse haplotypes, yellow; Isola Bella haplotypes)

was obtained using both the Community Climate System Model (CCSM) and the Model for Interdisciplinary Research on Climate (MIROC) available in the Worldclim database.

\section{Results}

\section{Phylogeographic structure and time of divergence}

The final $c y t b$ alignment was 935 bp $(n=277)$ from our samples and 765 bp $(n=76)$ from Podnar et al. (2005). The coalescent Bayesian phylogenetic analysis, inferred using BEAST and MrBayes, supported the same topology with seven well-supported clades (see Fig. 2a for supporting values at each node): T, A1, A2, A3, S1, S2 and $\mathrm{S} 3$. We found high genetic divergence among clades, with an overall mean distance $D_{\text {nei }}=0.055$, a maximum value between clade $T$ and clade $S 1\left(D_{\text {nei }}=0.092\right)$ and a minimum value between clade $\mathrm{A} 1$ and $\mathrm{A} 2\left(\mathrm{D}_{\text {nei }}=0.028\right)$. These seven clades were also supported by sPCA, performed on the whole mtDNA (see below).

Estimates of the TMRCAshowed an early separation at 2.59 Mya (95\% HPD: 2.78-2.39 Mya) into two main lineages, corresponding to the "Siculo-Calabrian" lineage and the "central-northern" lineage. Within the SiculoCalabrian lineage we identified three clades (S1, S2 and S3). The emergence of S1 occurred at 1.9 Mya (95\% HPD: 2.25-1.58 Mya) while S2 and S3 separated 1.54 Mya (95\% HPD: 1.88-1.2 Mya). The central-northern lineage split approximately 1.79 Mya (95\% HPD: 2.15$1.4 \mathrm{Mya}$ ) into two main groups that for simplicity we refer to as 'Adriatic'and 'Tyrrhenian'. The Adriatic group includes three clades with an early separation of the Croatian clade A1 at 1.22 Mya (95\% HPD: 1.58 Mya-890 Kya) followed by a subsequent separation between clade A2 and the northern Calabrian clade A3 at 812 Kya (95\% HPD: 1.03 Mya-552 Kya). See Fig. 2a for TMRCA at more internal nodes. We found a variable number of haplogroups within the seven clades. The haplogroups were defined by the high posterior probability support $(0.97-0.99)$ in the phylogenetic analysis and by their clustering in the Parsimony network (Fig. 2). The Tyrrhenian clade $\mathrm{T}(n=45, \mathrm{H}=25)$ is mainly distributed across the northern-central Tyrrhenian coast and includes three principal haplogroups $(\mathrm{Tb}, \mathrm{Tc}$ and $\mathrm{Td}$ ) with a south-north pattern and a single diverging haplotype (Ht28). The TMRCA for this clade was estimated at 846 Kya (95\% HPD: 1.14 Mya-573 Kya).

Clade A2 $(n=113, H=44)$ is the only clade we found along the Adriatic coast (excluding clade A1 which is restricted to the Curzolan Islands, Croatia). It is characterized by five haplogroups and two unique diverging haplotypes. Two haplogroups and the two diverging haplotypes are restricted to Campania and Basilicata (A2a, A2b, A2e and A2f), one haplogroup is from northern Calabria (A2g) and the remaining two are widespread across the Adriatic coast of Italy and Croatia. The A2c haplogroup has the northernmost gravitation while the A2d haplogroup is widespread towards the Italian and Croatian Adriatic coasts. Both are characterized by a star-like shape and very low haplotype diversity.

Clade A3 $(n=10, H=7)$ includes populations from northern coastal areas of Calabria (Catena Costiera). This clade is more related to the Adriatic clade A2, forming part of the central-northern lineage, than to the other clades found in southern Calabria.

Clades S1 $(n=22, H=18)$ and S2 $(n=6, H=6)$ represent the Calabrian "sensu stricto" lineages; in fact they are restricted to the Serre chain (and surrounding areas) and the Sila massif respectively. Clade S3 $(n=$ $152, H=101)$ groups haplotypes mainly belonging to Sicily. However, two southern Calabrian (Aspromonte massif) haplotypes (Hs98 and $\mathrm{Hs} 99$ ) also belong to this clade. They are separated by 10 and 13 substitutions from haplotypes found in the southernmost part of Sicily (Hyblean). Within Sicily, we identified 7 main haplogroups partially separated geographically (Fig. 2b-c) and a single divergent haplotype (Hs61) from Pachino. The oldest split within the clade was estimated at the Middle Pleistocene (95\% HPD: 800 Kya-460 Kya). The southernmost region (Hyblean) is characterized by haplogroups S3b and S3c and the divergent haplotype from Pachino. Western Sicily mainly includes a single haplogroup (S3h) with the exception of two divergent haplotypes (Hs26 and Hs27) from the Zingaro Nature Reserve and Monte Cofano (haplogroup S3e). Central Sicily includes a principal haplogroup (S3g), while the 
north-eastern region includes haplogroup S3i which also comprises individuals from the Aeolian Islands and haplogroup S3f with circum-Etnean gravitation.

Among the circum-Sicilian archipelagos, the Aegadian Islands of Marettimo and Favignana (blue bold in Fig. 2c) appear to be monophyletic, while the Aeolian Islands (violet bold in Fig. 2c) are polyphyletic and thus appear to have experienced independent colonization events. However, we did not find any shared Aeolian and Sicilian haplotypes. It is noteworthy that individuals from southern Corsica and from central and north-western Sardinia (green bold in the Fig. 2c) fall into the subhaplogroup present in the Aegadian Islands.

The sPCA, performed on the whole mtDNA, showed a stronger global than local structure $\left(P_{\text {global }}<0.001\right.$, $\left.P_{\text {local }}=0.9\right)$. When only PC1 and PC2 were considered, the sPCA supported the separation of five clades (T, A, S1, S2 and S3), while PC3 better explained the variation within the Adriatic clades A1, A2 and A3. The sPCA performed within each clade indicated significant global rather than local structure in T, A2, A3, S1 and S3 (T: $P_{\text {global }}=0.001, P_{\text {local }}=1 ; \mathrm{A} 2: P_{\text {global }}=0.001$, $P_{\text {local }}=1 ; \mathrm{A} 3: P_{\text {global }}=0.014, P_{\text {local }}=0.77 ;$ S3: $P_{\text {global }}=$ $\left.0.001, P_{\text {local }}=1\right)$, while clade A1 did not show a significant value of either global or local structure (A1: $P_{\text {glo }}$ bal $=0.36, P_{\text {local }}=0.61$ ) and clade S2 showed higher local than global structure.

The final nuclear DNA alignments were726 bp $(n=182)$ for $\beta$-fibint7, and $600 \mathrm{bp}(n=524)$ formc1r. No recombination events were found by the four-gamete and the phy test for both the nuclear genes.
We found 68 phased haplotypes for $\beta$-fibint7 connected in a single network which showed a certain structure matching to the main mtDNA clades (Fig. 3). Estimates of genetic diversity showed high level of nucleotide $(\pi=0.009 \pm 0.0005)$ and haplotype $(h=0.93$ $\pm 0.01)$ diversity, remarked also by the hierarchical AMOVA which indicated that $40.3 \%\left(\mathrm{~F}_{\mathrm{ct}}=0.39\right)$ of variation was among groups (i.e., mtDNA clades), $46 \%$ $\left(\mathrm{F}_{\mathrm{st}}=0.45\right)$ was within populations and $13.7 \%\left(\mathrm{~F}_{\mathrm{sc}}=0.13\right)$ was among populations within groups. The geographic allele distribution of the main $\beta$-fibint7 haplogroups is shown in Additional file 3: Figure S3.

The mc1r gene (consisting of 61 haplotypes) resulted less structured. The network connecting these haplotypes failed to identify clusters reflecting the lineages or the clades revealed by the mtDNA phylogenetic analysis (Fig. 3). Estimates of genetic diversity indicated lower values of nucleotide $(\pi=0.003 \pm 0.0001)$ and haplotype $(h=0.69 \pm 0.02)$ diversity compared to the $\beta$-fibint7. However, a certain level of geographic structure could be observed when the distribution of some haplotypes was taken into account (Additional file 4: Figure S4). We found the three most common haplotypes (M1, M2 and M34) present in $67 \%$ of the entire sample. They are connected to other low-frequency haplotypes in a star-like shape.

Haplotype M1 is widespread at high frequency in central Italy, Calabria and Sicily. M2 is found in all the studied area with the exception of Calabria. M34 is found in central-southern Italy and along the Adriatic coast, mirroring the distribution of the mtDNA 'Adriatic' (a) $m c 1 r$

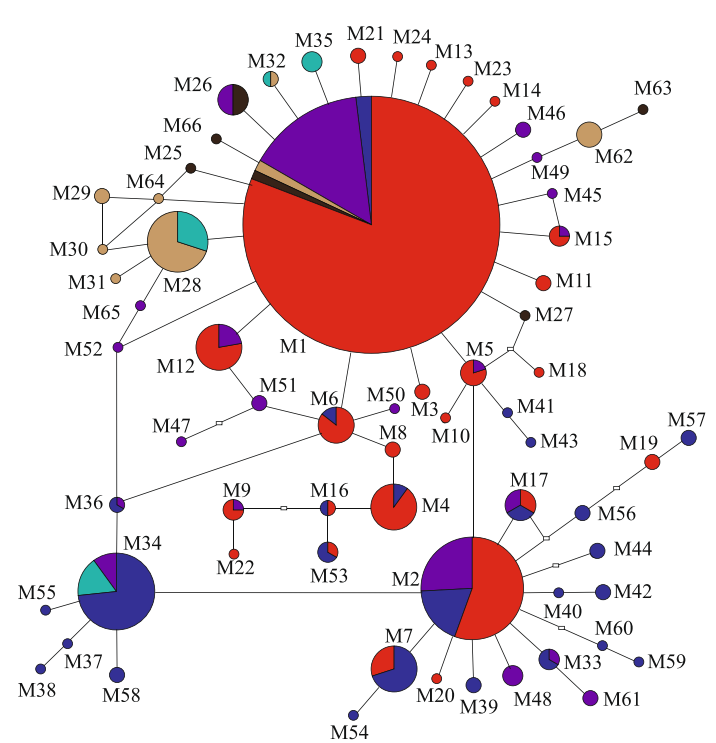

(b) $\beta$-fibint7

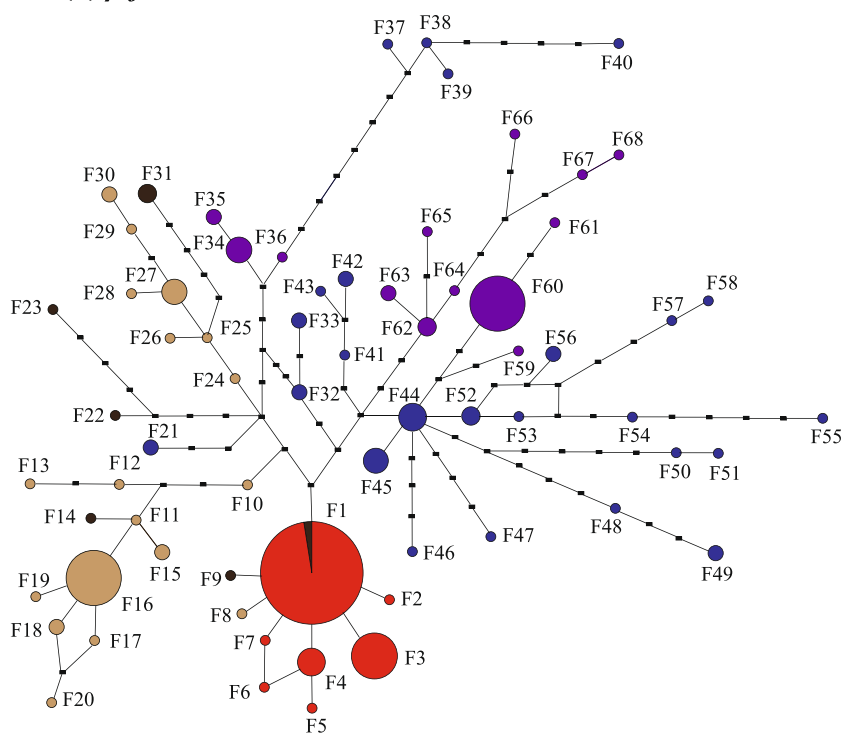

Fig. 3 Statistical parsimony network connecting the 61 mc1r haplotypes (a) and the $68 \beta$-fibint7 haplotypes (b). Each haplotype is represented by circles with size proportional to their frequencies. The colors depicts the identified mitochondrial clades 
clade. It is noteworthy that haplotype M1 was not found at all in this area. Finally, the analysis of molecular variance (AMOVA) carried out on mc1r using the grouping option revealed by the mtDNA clades indicated that $25.2 \%\left(\mathrm{~F}_{\mathrm{ct}}=0.87\right)$ of variation was among groups, $59 \%$ $\left(\mathrm{F}_{\mathrm{st}}=2.04\right)$ was within populations and $16 \%\left(\mathrm{~F}_{\mathrm{sc}}=0.55\right)$ was among populations within groups.

\section{Historical demography and spatial diffusion models}

The historical demography was performed on clades $\mathrm{T}$, A2, S1 and S3 which showed informative coalescent intervals in a time window of $0.6-0.4 \mathrm{Kya}$ (Fig. 4). The results of the Tajima's $D$ and Fu's $F s$ statistics are reported in Table 2.

The EBSP of the Tyrrhenian clade $\mathrm{T}$ showed prolonged demographic stability and a gradual pre-LGM expansion starting about $80 \mathrm{Kya}$ (Fig. 4a). The Adriatic clade A2 experienced a demographic expansion starting about 70 Kya (Fig. 4b). The EBSP of the Calabrian clade S1 showed prolonged stability followed by expansion occurred at the beginning of the last glacial phase (Fig. 4d). The Sicilian clade S3 experienced different demographic expansion events corresponding to the main glacial phases during the last 500 Kya (Fig. 4c).

The Bayesian phylogeography using continuous RRW summarized in three time slices identified the presence of several ancestral areas with 80\% HPDs at 2.3 Mya
(Fig. 5). The Siculo-Calabrian lineage showed four ancestral areas in Sicily and one in Calabria while the centralnorthern lineage showed a single ancestral area located in the Tyrrhenian coast of Tuscany. At 1.3 Mya, the $80 \%$ HPDs identified the emergence of additional ancestral areas especially throughout the Tyrrhenian coast (which correspond to the location of the main haplogroups), from which population expanded up to achieve a maximal spatial diffusion at about $0.1 \mathrm{Mya}$.

\section{Species distribution modelling}

Under current conditions (Fig. 6) the model predicts moderate and high suitability scores in coastal areas and in the southernmost regions but low habitat suitability in the whole Apennine mountain range and in northern regions. This fits very well with the known distribution of the species and its relative abundance.

Concerning the LGM prediction, high habitat suitability is maintained in coastal areas, especially throughout the Tyrrhenian coast and southern regions including a large area of high suitability in Sicily. Conversely, habitat suitability decreases in central and northern areas compared to the present-day distribution. The Adriatic Sea exhibited extensive land exposure due to the glacial marine regression. This area shows moderate to low habitat suitability along the coast and low habitat scores in internal areas.

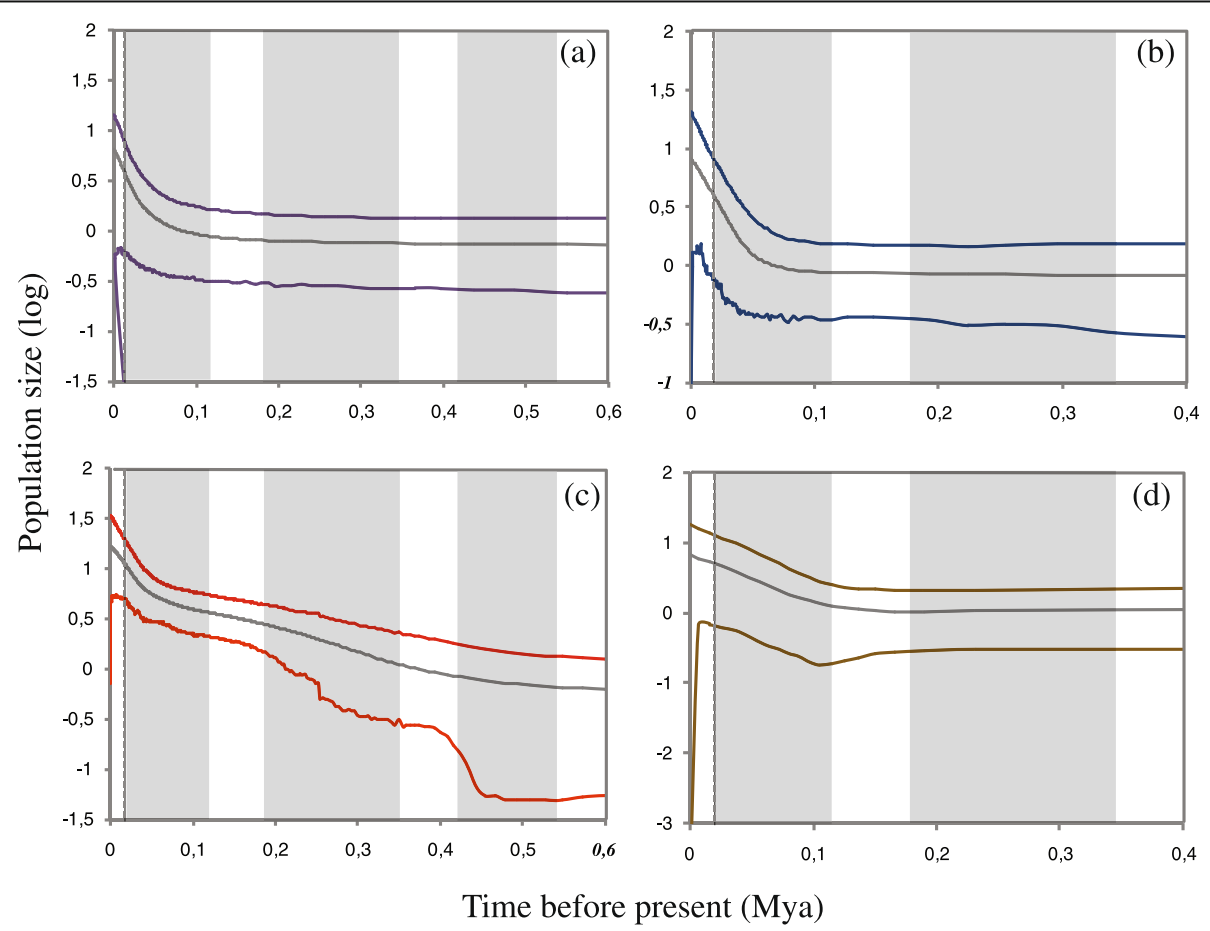

Fig. 4 Extended Bayesian skyline plots inferred with BEAST, illustrating effective population sizes (Ne) through time. The grey lines represents the median population size, and the colored lines represent 95\% higher posterior probability of (a) T clade, (b) A2 clade, (c) S3 clade and (d) S1 clade. The grey rectangles corresponds to the Würm, Riss and Mindel glacial periods while dashed lines indicate approximately the last glacial maximum 
Table 2 Mitochondrial (cytb) genetic diversity indices and neutrality tests for each clade

\begin{tabular}{lllllll}
\hline Clade & $n$ & $\mathrm{H}$ & $\mathrm{h} \pm \mathrm{SD}$ & $\pi \pm \mathrm{SD}$ & Tajima's D & Fu's Fs \\
\hline $\mathrm{T}$ & 45 & 25 & $0.94 \pm 0.02$ & $0.017 \pm 0.001$ & 0.27 & -2.58 \\
$\mathrm{~A} 1$ & 5 & 4 & $1.00 \pm 0.18$ & $0.004 \pm 0.001$ & $\backslash$ & $\backslash$ \\
$\mathrm{A} 2$ & 113 & 44 & $0.93 \pm 0.01$ & $0.0088 \pm 0.0003$ & $-1.45^{*}$ & $-20.34^{* *}$ \\
A3 & 10 & 7 & $0.96 \pm 0.04$ & $0.022 \pm 0.003$ & 0.13 & 0.136 \\
S1 & 22 & 18 & $0.97 \pm 0.02$ & $0.023 \pm 0.001$ & 0.65 & -2.36 \\
S2 & 6 & 6 & $0.93 \pm 0.12$ & $0.0045 \pm 0.001$ & $\backslash$ & $\backslash$ \\
S3 & 152 & 101 & $0.99 \pm 0.002$ & $0.0132 \pm 0.0005$ & $-1.76^{*}$ & $-84.9^{* *}$
\end{tabular}

MtDNA sample size $(n)$, number of haplotypes $(H)$, haplotype diversity with standard deviation $(h \pm S D)$, nucleotide diversity with standard deviation $(\pi \pm S D)$, Tajima's $D$ and Fu's $F s$ statistics for each identified clade ${ }^{*} P<0.05{ }^{* *} P<0.001$

\section{Discussion}

The phylogeographic analysis of the Italian wall lizard confirmed previous studies showing a strong mitochondrial phylogeographic structure with multiple lineages across its range (Fig. 2) [30]. However, the extended sampling across the Italian Peninsula and Sicily allowed the identification of surprising geographically nested lineage diversity, with additional haplogroups not identified previously, reflecting a more complex genetic pattern. The number of mtDNA lineages and their high level of divergence are, to the best of our knowledge, among the highest ever observed in any vertebrate species from the area. A similar high number of mtDNA lineages can be observed in the congeneric Podarcis muralis [28]. However, in this species the genetic divergences among lineages is much lower than the one observed in P. siculus.

This very high genetic partioning allowed an uncommonly small geographic scale dissection of the effect of Pleistocene climatic oscillation across an entire Mediterranean Pleistocene refugial area. This interpretation reflects at least two aspects of genetic complexity associated with different time-scale processes. The first deep phylogeographic divergence corresponds to major geographic barriers which produced prolonged allopatric differentiation during the Early Pleistocene (2.6-0.7 Mya). A second minor phylogeographic discontinuity is observed within clades, mirroring independent local differentiation in response to climatic fluctuation during the Early-Middle Pleistocene transition. Finally, short-term demographic expansions corresponding to the last glacial phase were also observed.

\section{Long-term allopatric fragmentation and maintenance of the parapatry of clades}

The structure of $P$. siculus mtDNA diversity into seven clades, with parapatric distribution, is strongly supported by both the phylogenetic analysis and SPCA, indicating significant global structure partioned into genetically distinguishable clusters.

Although the intrinsic inaccuracy of the molecular clock has been repeatedly reported ([59-62] and references therein), suggesting caution in the use of genetic data to infer dates of historical events, our approximate time window of long-term allopatric fragmentation appears to agree well with the major geomorphological events which characterized the main tectonic arrangements and eustatic events of the Italian Peninsula. The most ancient separation during the Pliocene-Pleistocene transition (approximately 2.78-2.39 Mya) matches the split of the southernmost Siculo-Calabrian lineage from the central-northern lineage. This result based on mtDNA is in accordance with an allozyme analysis at twenty loci [63]. The location of this genetic discontinuity reflects the specific position of the Crati-Sibari plain in northern Calabria. Indeed, this area acted as a dispersion barrier during the repeated Plio-Pleistocene marine transgressions separating the Italian Peninsula from the Calabrian and Sicilian paleo-archipelagos $[64,65]$. This main vicariance event has also been observed in other detailed works on different vertebrate taxa, confirming this region as one of the most important in driving long-term allopatric differentiation in southern Italy $[25,66-69]$.

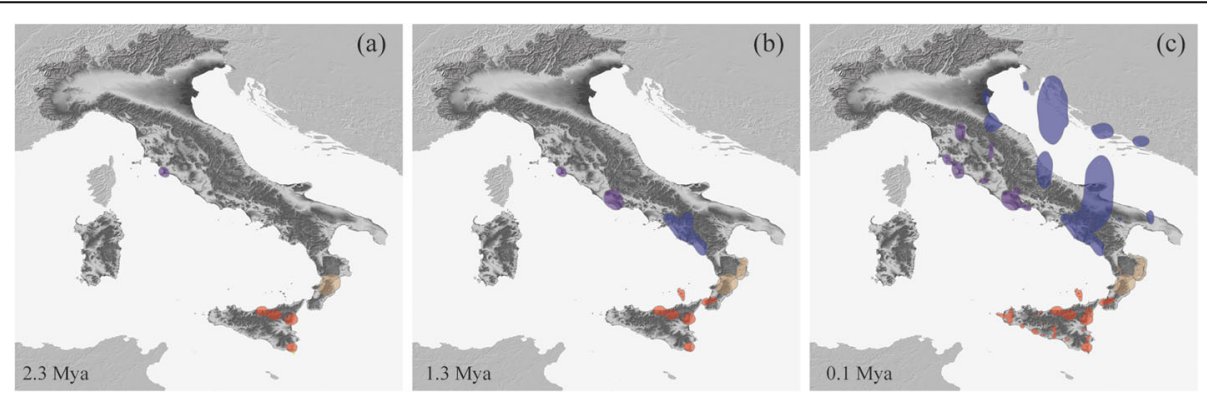

Fig. 5 Estimates of spatial diffusion of Podarcis siculus using mtDNA data set at three time points during the Pleistocene (a) 2.3 Mya, (b) 1.3 Mya, (c) $0.1 \mathrm{Mya}$. The polygons are colored according to each clade and indicate the uncertainty (80\% HPDs) surrounding geographic locations of internal nodes of the MCC tree 


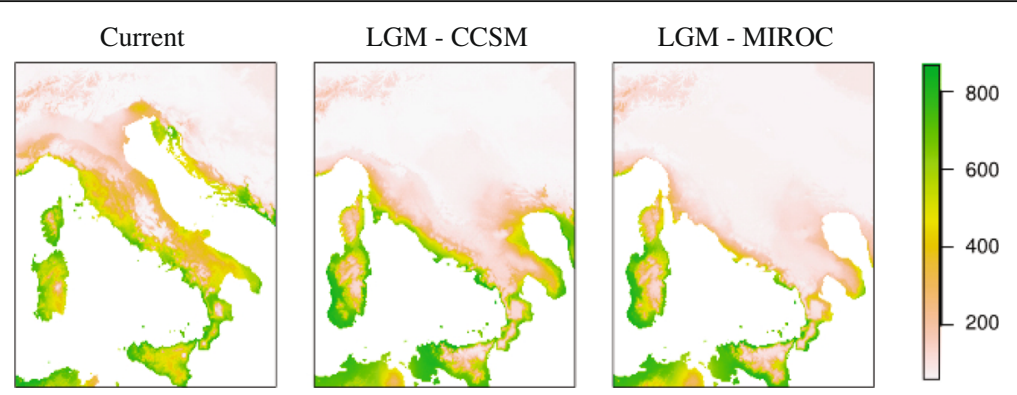

Fig. 6 Species distribution model for Podarcis siculus indicating the present-day conditions and the last glacial maximum (LGM) based on the Model for Interdisciplinary Research on Climate (MIROC) and Community Climate System Model (CCSM)

The more recent split of the three clades within the Siculo-Calabrian lineage, dated approximately 1.54-1.9 Mya, probably reflects the Early Pleistocene fragmentation of the Calabrian arc into paleo-islands corresponding to the Serre and Sila massifs and Aspromonte. In fact, clade $\mathrm{S} 1$ is found in the Sila massif and surrounding areas, while S2 is mainly distributed across the Serre region. It is interesting to note that an overlapping phylogeographic structure has been reported in the region for the Italian newt, Lissotriton italicus and the Roman mole, Talpa romana $[25,69]$.

The presence of an ancient S3 clade mostly distributed throughout Sicily is not straightforward, having few paralellisms in the literature [70-72]. This continental island experienced recurrent connection with the mainland during the glacial phases. It should be pointed out that the S3 clade and some sicilian $\beta$ fibint 7 haplotypes were also found in the southernmost part of Calabria. According to the BPA this occurrence suggested an ancient backward recolonization event probably due to land bridge connections during the Gunz-Mindel transition. The more ancient Middle Pleistocene split within Sicilian haplogroups agrees geographically and temporally with the presence of the Hyblean and Peloritani land masses, which were separated by the Gela-Catania channel until the more recent emergence of the Etna Volcano [73, 74]. This dichotomy is also evident in the position of the ancestral areas in Sicily depicted by the BPA (Fig. 5a).

Deep vicariance events during the Early-Middle Pleistocene (2.25-1.58 Mya) were also observed between the $\mathrm{T}$ and $\mathrm{A} 2$ clades within the central-northern lineage. The geographic boundary observed between the Tyrrenian clade $\mathrm{T}$ and the Adriatic clade A2, whose split is dated at 1.9 Mya (Early Pleistocene), has been reported in various taxa $[20,28,75]$. The genetic discontinuites seem to be located in different geographic positions. The south-north boundary corresponds to a well-known deep phylogeographic discontinuity matching the Volturno plain. This area was repeatedly flooded by Middle Pleistocene marine transgressions, thus constituting an effective geographic barrier between previously separated populations [76, 77]. Conversely, the west-east boundary seems to be the outcome of a recent recolonization route of the $\mathrm{A} 2 \mathrm{~d}$ haplogroup from the southernmost regions (see below), confirming the role of the Apennine range as a longitudinal diffusion barrier.

While the $\beta$-fibint7 showed evidence of genetic discontinuity according to the mitochondrial signal, the $m c 1 r$ resulted in a lower phylogeographic structure. The occurrence of incomplete lineage sorting to explain mitonuclear discordance has been describer for an increasing number of case studies [78-80]. Thus, the failure of nuclear genome to diverge as a consequence of its slower diverging rate and higher population size appear the most plausible interpretation to explain such pattern rather than sex-biased gene flow [81-83]. Moreover, the higher number of retained polymorphisms in the $m c 1 r$ marker is also consistent with the more relaxed rate of lineage sorting for coding genes which therefore could be under selection [84-86]. However, the two nuclear genes also showed a certain degree of consistency regarding the locations of the principal genetic discontinuities (Additional file 3: Figure S3 and Additional file 4: Figure S4). This is remarked by the high percentage, for both genes, of the among groups variation $(40.3 \%$ for $\beta$ fibint7 and $25.2 \%$ for $m c 1 r$ ) revealed by the AMOVA, hierarchically defined by the mtDNA clades.

One of the main questions emerging from this study is how such close parapatric structure between mtDNA clades, as seen in Fig. 2b, could be maintained. This pattern is also supported by the complete absence (out 59 localities with $2<\mathrm{N}<10$ ) of the co-occurrence of mtDNA clades in the same locality, even though the outcome of repeated cycles of allopatric fragmentation and local expansion would give rise to a certain admixture of genetic lineages. This phenomenon is only now becoming better known $[87,88]$ and the main explanations include "density blocking", local adaptation (such as adaptation of the mtDNA genome) and competitive exclusions. In this case-study, we cannot exclude a certain level of hybrid unfitness [87]. In fact, the level of 
genetic divergence at $c y t b$ is only slightly lower than that commonly found between sister species in Podarcis [42, 89, 90].

Further detailed studies are required in the contact areas of the mtDNA clades, including estimates of gene flow using more variable markers (i.e. microsatellites) and detailed analyses of the sexual selection and other eco-ethological characteristics of these differentiated interacting populations [91, 92].

\section{Dissecting the evolutionary history and demography of the clades}

The evolutionary history of the clades appears to have been much more influenced by a series of historical fragmentations due to severe cycles of glacial/interglacial periods during the Early-Middle Pleistocene than by recent demographic responses to the LGM. However, we also found independent responses of each clade to such longterm and short-term processes.

The main minor genetic discontinuities within clades are traceable to a time window corresponding to the Early-Middle Pleistocene transition (1.3-0.7 Mya) [93]. The latter subepoch transition, also known as the 'MidPleistocene Revolution' [94], is associated with a series of complex climatic changes leading to a more severe cold stage [95]. The main outcome of the increasing amplitude in climatic fluctuations is the more drastic responses of temperate species in retreating into suboptimal refugial areas. This phenomenon is well exemplified by clades $\mathrm{S} 2$ and $\mathrm{T}$. The separation of the two main haplogroups in clade $\mathrm{S} 2$ reflects two possible refugial areas corresponding to the northern and southern areas surrounding the Sila massif, which finds some parallelisms in the literature $[25,69]$. The BPA identified two putative refugial areas, well apart along the Tyrrhenian coast, for the haplogroups within the clade $T$. It is noteworthy that the current distribution of the two main haplogroups (Tc and Td) across this central peninsular region appears very similar to that observed in the southern smooth newt Lissotriton vulgaris meridionalis [29].

A different scenario emerges from examination of the genetic pattern in Sicily (clade S3). Since there are no substantial geographic barriers across the entire island, the strong phylogeographic structure, with seven haplogroups, is quite surprising and provides the highest resolution to date for the structure of Sicilian phylogeographic diversity [72]. At least two considerations deserve attention regarding this insular context. First, the temporal incongruence of the intra-island diversification (late Middle Pleistocene) with the Early-Middle Pleistocene transition could be the outcome of attenuated or shifted effects of the Pleistocene oscillation throughout this southernmost Mediterranean island. In any case, the perception that Sicily has experienced reduced climatedriven environmental changes is also evident by comparing current and past SDM. Second, the low variation of the nuclear markers suggests that these haplogroups would not have had completely independent evolutionary histories, maintaining a long-term mutation drift equilibrium. This is also confirmed by the SDM indicating different stable connected areas that may have guaranteed this gene exchange and equilibrium.

The cradle of clade A2 appears to be in the southern part of its distribution, as indicated by the BPA, where five of the seven haplogroups are located. Within this clade we found a combination of ancient isolation and more recent demographic dynamics. The main genetic discontinuities are restricted to southern-central Italy and may reflect Middle Pleistocene fragmentation due to the complex topography of this area. However, haplogroups A2d and A2c show a north-eastern gravitation, being the only haplogroups found on the Adriatic coast and in the Po Valley. The demographic inference for this clade, supported by the BPA, highlights an overall population expansion during the last glacial phase (about $70 \mathrm{Kya}$ ) from localities settled in the southern Tyrrhenian coast. Thus the presence of this clade throughout the Croatian coast is not very surprising considering the extended Adriatic land exposure during this period [96]. A similar glacial Adriatic expansion has also been proposed for Hyla intermedia [66]. Furthermore, such glacial colonization would have taken place from just a few pioneers, as suggested by the very low genetic diversity encountered and the overall low LGM habitat suitability predicted for this area.

Finally, while a vast body of literature clearly demonstrates that many terrestrial species underwent large and sudden latitudinal shifts following the end of the last glacial maximum (LGM) approximately 20 Kya ([1, 3, 97] and refs therein), we found a different scenario consistent with glacial expansions or regional persistence during the LGM. The hypothesis of glacial expansion and local persistence, reported in a growing number of Palearctic and Nearctic species $[28,98,99]$, was criticized for the possible inaccuracy of molecular clock rates [100, 101]. However, our claim is supported not only by the estimated time of divergence but also by the evidence of large amount of new environment with high suitability, predicted by SDM, which appear during LGM in different southern Italian regions. This is particularly evident for the Sicilian clade S3. Indeed our demographic scenario indicates two expansions corresponding to the main glacial phases at 400 Kya and $100 \mathrm{Kya}$. As a matter of fact, the SDM prediction at LGM shows that Sicily is the area where the effect of the increase of high suitability areas and lowland exposure is most prominent for the entire species range. Although the glacial expansions in T and S2 were of smaller strength, 
these areas were also influenced by the increase of the coastal plain during the last glacial phase.

\section{Conclusions}

\section{The exception became the rule?}

The main outcomes of this study show that the classic EC model Pleistocene biogeography may be a far too simplistic interpretation of the evolutionary history of $P$. siculus. The high number of deeply divergent clades matches a 'refugia within refugia' scenario [1] rather than a single panmictic refugium for the Italian Peninsula. However, despite the greater number of clades located in southern regions as expected from the "southern richness" scenario $[1,2,102]$, our results showed a genetic structure conforming more to a pattern that here can be called "western richness and eastern purity". This scheme also appears to be well supported by the current and past SDM predictions and location of ancestral areas indicated by BPA. In fact, the Tyrrhenian coastline appears to have maintained more stable climatic conditions than the Adriatic one. Hence, the southernmost and Tyrrhenian regions represent hot pockets in which populations may have preserved their deep genetic integrity by persisting in scattered refugial areas during strong Pleistocene cooling periods, from which they later could have expanded towards new areas. We did not find evidence of expansion subsequent to the LGM in any of the analyzed clades. Instead we found genetic signatures of demographic expansion preceding the LGM or long periods of stability. This complex assembly of genetic signatures appears to be distant from the expectation of the EC models and post-LGM recolonizations, becoming one of an increasing number of cases in which these assumptions are not met.

The historical demography coupled with the evolutionary history reconstruction indicated that long-term fragmentation and/or pre-LGM events such as glacial persistence were more important in shaping the genetic architecture in this temperate species. Such historical events may have influenced populations to different extents, indicating the importance of local topographic impacts on Quaternary climatic oscillations in inducing population-specific responses to the ever changing environmental variability.

\section{Additional files}

Additional file 1: Table S1. Sample size of each locality (with relative locality codes). MtDNA and nuDNA haplotypes are also reported for each sampling location. (DOCX $54 \mathrm{~kb}$ )

Additional file 2: Table S2. Voucher with relative accesison numbers and haplotypes. (DOCX $58 \mathrm{~kb}$ )

Additional file 3: Figure S3. Geographic allele distribution of $\beta$-fibint7 with relative frequencies indicated by pie diagrams at each sampled location. (JPG $4081 \mathrm{~kb}$ )
Additional file 4: Figure S4. Geographic allele distribution of $\mathrm{mclr}$ with relative frequencies indicated by pie diagrams at each sampled location. (JPG $4161 \mathrm{~kb})$

\section{Abbreviations}

AIC: Akaike information criterion; AUC: Area under the curve; BIC: Bayesian information criterion; BPA: Bayesian phylogeographic analysis; CCSM: Community climate system model; EBSP: Extended Bayesian Skyline Plot; EC: Expansioncontraction; GAM: General additive model; GBIF: Global biodiversity information system; GLM: Generalized linear model; HPD: Highest posterior distribution; IUPAC: International union of pure and applied chemistry; Kya: Thousand $\left(10^{3}\right)$ years ago; LGM: Last glacial maximum; MCC: Maximum clade credibility; MCMC: Markov chain Monte Carlo; MIROC: Model for interdisciplinary research on climate; mtDNA: Mitochondrial DNA; Mya: Million $\left(10^{6}\right)$ years ago; nuDNA: Nuclear DNA; PCA: Principal component analysis; RRW: Relaxed random walk;

SDM: Species distribution modelling; TMRCA: Time of the most recent common ancestor; TSS: True skill statistic

\section{Acknowledgements}

The authors are very grateful to all the collectors who have contributed to improving our sampling. Thanks are also extended to Flavia Annesi and Ignazio Avella for their assistance in the laboratory. We wish to address a special thanks to Dario D' Eustacchio, tragically passed away, for his precious suggestions and for his valuable help in the field. Finally we want to thanks the two anonymous reviewers for their appreciated comments.

\section{Funding}

This study was supported by the Italian Ministry of Education, University and Research (PRIN 2012 project) and by "Progetti di Ricerca di Università" to RC.

\section{Availability of data and materials}

mtDNA and nuDNA sequences are available in GenBank (accession numbers from KY064841 to KY065117 for cytb, from KY064579 to KY064730 for mc1r and from KY094514 to KY094604 for $\beta$-fibint7).

Climate data and geographic references will be submitted to Dryad upon acceptance.

\section{Authors'contributions}

Conceived and designed the study: GS, RC. Performed the field work: GS, GA, ED, RC. Laboratory procedures: GS, ED. Data analysis: GS, PC, RC Wrote the paper: GS. All authors read and approved the final manuscript.

\section{Competing interests}

The authors declare that they have no competing interests.

\section{Consent for publication}

Not applicable.

\section{Ethics approval and consent to partecipate}

All the tissue samples used in this work were collected with permission of the Italian Environment Ministry for the Environment, Land and Sea to RC. (Prot. 00017879/PNM del 09/09/2012) and no animal was killed.

\section{Author details}

'Dipartimento di Biologia e Biotecnologie "Charles Darwin", Università di Roma LA SAPIENZA, sede di Anatomia comparata, Rome, Italy. ${ }^{2}$ National Research Council, Institute of Ecosystem Study, Largo Tonnoli 50, 28922 Verbania Pallanza, Italy. ${ }^{3}$ Museo di Storia Naturale della Calabria e Orto Botanico, Università della Calabria, CAP 87036 Rende, Cosenza, Italy.

Received: 5 July 2016 Accepted: 8 December 2016

Published online: 05 January 2017

\section{References}

1. Hewitt GM. Some genetic consequences of ice ages and their role in divergence and speciation. Biol J Linn Soc. 1996;58:247-76.

2. Hewitt GM. The genetic legacy of the Quaternary ice ages. Nature. 2000;405:907-13.

3. Schmitt T. Molecular biogeography of Europe: pleistocene cycles and postglacial trends. Front Zool. 2007:4:11. 
4. Provan J, Bennet KD. Phylogeographic insights into cryptic glacial refugia. Trends Ecol Evol. 2008;23:564-71.

5. Hickerson MJ, Carstens BC, Cavender-Bares J, Crandall KA, Graham CH, Johnson JB, Yoder AD. Phylogeography's past, present, and future: 10 years after. Mol Phylogenet Evol. 2010;54:291-301.

6. Hengeveld R. Ecological biogeography. Prog Phys Geogr. 1993;17:448-60.

7. Taberlet P, Fumagalli LA, Wust-Saucy AG, Cosson JF. Comparative phylogeography and postglacial colonization routes in Europe. Mol Ecol. 1998;7:453-64.

8. Stewart JR. The progressive effect of the individualistic response of species to Quaternary climate change: an analysis of British mammalian faunas. Quat Sci Rev. 2008;27:2499-508.

9. Stewart JR, Lister AM, Barnes I, Dalén L. Refugia revisited: individualistic responses of species in space and time. Proc R Soc London, Ser B. 2010;277:661-71.

10. Holderegger R, Thiel-Egenter C. A discussion of different types of glacial refugia used in mountain biogeography and phylogeography. J Biogeogr. 2009;36:476-80.

11. Hardion L, Baumel A, Verlaque R, Vila B. Distinct evolutionary histories of lowland biota on Italian and Balkan peninsulas revealed by the phylogeography of Arundoplinii (Poaceae). J Biogeogr. 2014;41:2150-61.

12. Alberdi A, Gilbert MTP, Razgour O, Aizpurua O, Aihartza J, Garin I. Contrasting population-level responses to Pleistocene climatic oscillations in an alpine bat revealed by complete mitochondrial genomes and evolutionary history inference. J Biogeogr. 2015;42:1689-700.

13. Bisconti R, Canestrelli D, Colangelo P, Nascetti G. Multiple lines of evidence for demographic and range expansion of a temperate species (Hyla sarda) during the last glaciation. Mol Ecol. 2011;20:5313-27.

14. Salvi D, Schembri PJ, Sciberras A, Harris DJ. Evolutionary history of the Maltese wall lizard Podarcisfilfolensis: insights on the 'ExpansionContraction'model of Pleistocene biogeography. Mol Ecol. 2014;23:1167-87.

15. Gomez A, Lunt DH. Refugia within refugia: patterns of phylogeographic concordance in the Iberian Peninsula. In: Phylogeography in Southern European Refugia: evolutionary perspectives on the origins and conservation of European Biodiversity. Springer; 2007. p. 155-188.

16. Dubreuil M, Riba M, Mayol M. Genetic structure and diversity in Ramondamyconi (Gesneriaceae): effects of historical climate change on a preglacial relict species. Am J Bot. 2008;95:577-87.

17. Centeno-Cuadros A, Delibes M, Godoy JA. Phylogeography of Southern Water Vole (Arvicolasapidus): evidence for refugia within the Iberian glacial refugium? Mol Ecol. 2009;18:3652-67.

18. Ferrero M, Blanco-Aguiar JA, Lougheed SC, Sanchez-Barbudo I, De Nova PJ, Davila JA. Phylogeography and genetic structure of the red-legged partridge (Alectorisrufa): more evidence for refugia within the Iberian glacial refugium. Mol Ecol. 2011;20:2628-42.

19. Canestrelli D, Nascetti G. Phylogeography of the pool frog Rana (Pelophylax) lessonae in the Italian peninsula and Sicily: multiple refugia, glacial expansions and nuclear-mitochondrial discordance. J Biogeogr. 2008;35:1923-36.

20. Canestrelli D, Bisconti R, Nascetti G. Extensive unidirectional introgression between two salamander lineages of ancient divergence and its evolutionary implications. Sci Rep. 2014;4:6516.

21. Ursenbacher S, Schweiger S, Tomovic L, et al. Molecular phylogeny of the nose-horned viper (Viperaammodytes, Linnaeus (1758)): evidence for high genetic diversity and multiple refugia in the Balkan peninsula. Mol Phylogenet Evol. 2008;46:1116-28.

22. Pabijan M, Zieliński P, Dudek K, Chloupek M, Sotiropoulos K, Liana M, Babik $W$. The dissection of a Pleistocene refugium: phylogeography of the smooth newt, Lissotriton vulgaris, in the Balkans. J Biogeogr. 2015;42:671-83.

23. Weiss S, Ferrand N. Current perspectives in phylogeography and the significance of South European refugia in the creation and maintenance of European biodiversity. In: Phylogeography of Southern European refugia. Springer Netherlands; 2007. p. 341-57.

24. Schmitt T, Varga Z. Extra-Mediterranean refugia: the rule and not the exception. Front Zool. 2012;9:1742-9994.

25. Canestrelli D, Aloise G, Cecchetti S, Nascetti G. Birth of a hotspot of intraspecific genetic diversity: notes from the underground. Mol Ecol. 2010;19:5432-51.

26. Bonfiglio L, Mangano G, Marra AC, Masini F, Pavia M, Petruso D. Pleistocene calabrian and sicilian bioprovinces. Geobios. 2002;35:29-39.

27. Marra AC. Pleistocene mammal faunas of Calabria (Southern Italy): biochronology and palaeobiogeography. Boll Soc Paleontol Ital. 2009;48:2.
28. Salvi D, Harris DJ, Kaliontzopoulou A, et al. Persistence across Pleistocene ice ages in Mediterranean and extra-Mediterranean refugia: phylogeographic insights from the common wall lizard. BMC Evol Biol. 2013;13:147.

29. Maura M, Salvi D, Bologna MA, Nascetti G, Canestrelli D. Northern richness and cryptic refugia: phylogeography of the Italian smooth newt Lissotriton vulgaris meridionalis. Biol J Linn Soc. 2014;113:590-603.

30. Podnar M, Mayer W, Tvrtkovic N. Phylogeography of the Italian wall lizard, Podarcis siculus, as revealed by mitochondrial DNA sequences. Mol Ecol. 2005; 14:575-88.

31. Salah MA, Martinez I. Universal and rapid salt-extraction of high quality genomic DNA for PCR-based techniques. Nucleic Acids Res. 1997;25:4692-3.

32. Podnar M, Haring E, Pinsker W, Mayer W. Unusual origin of a nuclear pseudogene in the Italian wall lizard: intergenomic and interspecific transfer of a large section of the mitochondrial genome in the genus Podarcis (Lacertidae). J Mol Evol. 2007;64:308-20.

33. Stephens M, Smith NJ, Donnelly P. A new statistical method for haplotype reconstruction from population data. Am J Hum Genet. 2001;68:978-89.

34. Stephens M, Donnelly P. A comparison of bayesian methods for haplotype reconstruction from population genotype data. Am J Hum Genet. 2003;73:1162-9.

35. Librado P, Rozas J. DnaSP v5: a software for comprehensive analysis of DNA polymorphism data. Bioinformatics. 2009;25:1451-2.

36. Flot JF, Tillier A, Samadi S, Tillier S. Phase determination from direct sequencing of length-variable DNA regions. Mol Ecol. 2006;6:627-630.37.

37. Tamura K, Peterson D, Peterson N, Stecher G, Nei M, Kumar S. MEGA5: molecular evolutionary genetics analysis using maximum likelihood, evolutionary distance, and maximum parsimony methods. Mol Biol Evol. 2011;28:2731-2739.38.

38. Huson DH, Bryant D. Application of phylogenetic networks in evolutionary studies. Mol Biol Evol. 2006;23(2):254-67.

39. Darriba D, Taboada GL, Doallo R, Posada D. jModelTest 2: more models, new heuristics and parallel computing. Nat Methods. 2012;9:772-2.

40. Drummond AJ, Suchard MA, Xie D, Rambaut A. Bayesian phylogenetics with BEAUti and the BEAST 1.7. Mol Biol Evol. 2012;29:1969-73.

41. Ronquist F, Teslenko M, Van der Mark P, Ayres DL, Darling A, Höhna S, Huelsenbeck JP. MrBayes 3.2: efficient Bayesian phylogenetic inference and model choice across a large model space. Syst Biol. 2012;61:539-42.

42. Poulakakis N, Lymberakis P, Antoniou A, et al. Molecular phylogeny and biogeography of the wall-lizard Podarcis erhardii (Squamata: Lacertidae). Mol Phylogenet Evol. 2003;28:38-46.

43. Harris DJ, Sá-Sousa P. Molecular phylogenetics of Iberian wall lizards (Podarcis): is Podarcis hispanica a species complex? Mol Phylogenet Evol. 2002:23:75-81.

44. Rambaut A, Suchard MA, Xie D, Drummond AJ. Tracer v1.6. 2014. Available from http://beast.bio.ed.ac.uk/Tracer.

45. Clement M, Posada D, Crandall KA. TCS: a computer program to estimate gene genealogies. Mol Ecol. 2000;9:1657-9.

46. Jombart $\mathrm{T}$. adegenet: a R package for the multivariate analysis of genetic markers. Bioinformatics. 2008;24:1403-5.

47. Dellicour S, Mardulyn P. SPADS 1.0: a toolbox to perform spatial analyses on DNA sequence data sets. Mol Ecol Resour. 2014;14:647-51.

48. Ho SY, Shapiro B. Skyline-plot methods for estimating demographic history from nucleotide sequences. Mol Ecol Resour. 2011;11:423-34.

49. Minin VN, Bloomquist EW, Suchard MA. Smooth skyride through a rough skyline: Bayesian coalescent-based inference of population dynamics. Mol Biol Evol. 2008;25(7):1459-71.

50. Lemey P, Rambaut A, Welch JJ, Suchard MA. Phylogeography takes a relaxed random walk in continuous space and time. Mol Biol Evol. 2010;27:1877-85.

51. Bielejec F, Rambaut A, Suchard MA, Lemey P. SPREAD: spatial phylogenetic reconstruction of evolutionary dynamics. Bioinformatics. 2011;27(20):2910-2.

52. Hijmans RJ, Cameron SE, Parra JL, Jones PG, Jarvis A. Very high resolution interpolated climate surfaces for global land areas. Int I Climatol. 2005:25:1965-78.

53. Thuiller W, Lafourcade B, Engler R, Araújo MB. BIOMOD-a platform for ensemble forecasting of species distributions. Ecography. 2009;32:369-73.

54. Phillips SJ, Anderson RP, Schapire RE. Maximum entropy modeling of species geographic distributions. Ecol Model. 2006;190:231-59.

55. Phillips SJ, Dudík M. Modeling of species distributions with Maxent: new extensions and a comprehensive evaluation. Ecography. 2008;31:161-75.

56. Ridgeway G. The state of boosting. Comput Sci Stat. 1999;31:172-81. 
57. McCullagh P, Nelder JA. Generalized linear models. Florida: Chapman and Hall/CRC, Boca Raton; 1989

58. Hastie TJ, Tibshirani RJ. Generalized additive models (Vol. 43). CRC Press; 1990

59. Ayala FJ. Vagaries of the molecular clock. Proc Natl Acad Sci U S A. 1997;94:7776-83.

60. Ayala FJ. Molecular clock mirages. Primates. 1997:8:9

61. Gibbons A. Calibrating the mitochondrial clock. Science. 1998;279:28-9.

62. Burridge CP, Craw D, Fletcher D, Waters JM. Geological dates and molecular rates: fish DNA sheds light on time dependency. Mol Biol Evol. 2008;25:624-33.

63. Capula M, Ceccarelli A. Distribution of genetic variation and taxonomy of insular and mainland populations of the Italian wall lizard, Podarcis siculus. Amphib-Reptilia. 2003;24:483-95.

64. Martini IP, Sagri M, Colella A. Neogene-quaternary basins of the inner Apennines and Calabrian arc. In: Anatomy of an Orogen: the Apennines and Adjacent Mediterranean Basins. Netherlands: Springer; 2001. p. 375-99.

65. Cucci L. Raised marine terraces in the Northern Calabrian Arc (Southern Italy): a 600 kyr-long geological record of regional uplift. Ann Geophys. 2004;47(4): 1391-406.

66. Canestrelli D, Cimmaruta R, Nascetti G. Phylogeography and historical demography of the Italian tree frog Hyla intermedia reveals multiple refugia, population expansions and secondary contacts within peninsular Italy. Mol Ecol. 2007;16:4808-21.

67. Grill A, Amori G, Aloise G, Lisi I, Tosi G, Wauters LA, Randi E. Molecular phylogeography of European Sciurus vulgaris: refuge within refugia? Mol Ecol. 2009;18:2687-99.

68. Colangelo P, Aloise G, Franchini P, Annesi F, Amori G. Mitochondrial DNA reveals hidden diversity and an ancestral lineage of the bank vole in the Italian peninsula. J Zool. 2012;287:41-52.

69. Canestrelli D, Sacco F, Nascetti G. On glacial refugia, genetic diversity, and microevolutionary processes: deep phylogeographical structure in the endemic newt Lissotriton italicus. Biol J Linnean Soc. 2012;105:42-55.

70. Seddon JM, Santucci F, Reeve NJ, Hewitt GM. DNA footprints of European hedgehogs, Erinaceus europaeus and E. concolor: Pleistocene refugia, postglacial expansion and colonization routes. Mol Ecol. 2001;10:2187-98.

71. Bezerra AM, Annesi F, Aloise G, Amori G, Giustini L, Castiglia R. Integrative taxonomy of the Italian pine voles, Microtus savii group (Cricetidae, Arvicolinae). Zool Scr. 2015;45:225-36.

72. Stöck M, Griffoni G, Amor N, Scheidt U, Sicilia A, Novarini N. On the origin of the recent herpetofauna of Sicily: comparative phylogeography using homologous mitochondrial and nuclear genes. Zool Anz. 2016. doi:10.1016/ j.jcz.2015.10.005.

73. Bonfiglio L, Burgio E. Significato paleoambientale e cronologico delle mammalofaune pleistoceniche della Sicilia in relazione all'evoluzione paleogeografica. II Quaternario. 1992;5:223-34

74. Stroscio S, Baviera C, Frati F, Lo Paro G, Nardi F. Deep genetic divergence in the darkling beetle Pimelia rugulosa (Coleoptera, Tenebrionidae) reflects Plio-Pleistocenic paleogeographic history of Sicily. J Zool Sys Evol Res. 2011:49:196-203.

75. Mezzasalma M, Dall'Asta A, Loy A, Cheylan M, Lymberakis P, Zuffi MA, Guarino FM. A sisters' story: comparative phylogeography and taxonomy of Hierophis viridiflavus and H. gemonensis (Serpentes, Colubridae). Zool Scr. 2015:44(5):495-508.

76. Romano P, Santo A, Voltaggio M. Evoluzione geomorfologica della piana del fiume Volturno (Campania) durante il tardo Quaternario (Pleistocene medio-superiore - Olocene). II Quaternario. 1994;7:41-56.

77. Barra D, Romano P, Santo A, Campajola L, Roca V, Tuniz C. The versilian transgression in the Volturno River plain (Campania, Italy): palaeoenvironmental evolution and chronological data. II Quaternario. 1996;9:445-58.

78. McGuire JA, Linkem CW, Koo MS, Hutchison DW, Lappin AK, Orange DI, Jaeger JR. Mitochondrial introgression and incomplete lineage sorting through space and time: phylogenetics of crotaphytid lizards. Evolution. 2007;61(12):2879-97

79. Toews DP, Brelsford A. The biogeography of mitochondrial and nuclear discordance in animals. Mol Ecol. 2012;21(16):3907-30.

80. De Biasse MB, Nelson BJ, Hellberg ME. Evaluating summary statistics used to test for incomplete lineage sorting: mito-nuclear discordance in the reef sponge Callyspongia vaginalis. Mol Ecol. 2014;23(1):225-38.

81. Ujvari B, Dowton M, Madsen T. Population genetic structure, gene flow and sex-biased dispersal in frillneck lizards (Chlamydosaurus kingii). Mol Ecol. 2008;17(15):3557-64.
82. Beysard M, Perrin N, Jaarola M, Heckel G, Vogel P. Asymmetric and differential gene introgression at a contact zone between two highly divergent lineages of field voles (Microtus agrestis). J Evol Biol. 2012;25(2):400-8.

83. Irwin DE. Phylogeographic breaks without geographic barriers to gene flow. Evolution. 2002;56:2383-94.

84. Rosenblum EB, Hoekstra HE, Nachman MW. Adaptive reptile color variation and the evolution of the MCIR gene. Evolution. 2004;58(8):1794-808.

85. Rosenblum EB, Römpler H, Schöneberg T, Hoekstra HE. Molecular and functional basis of phenotypic convergence in white lizards at White Sands. Proc Natl Acad Sci. 2010;107(5):2113-7.

86. Olsson M, Stuart-Fox D, Ballen C. Genetics and evolution of colour patterns in reptiles. In: Seminars in cell \& developmental biology. Academic Press; 2013, July;24(6), pp. 529-541.

87. Waters JM, Fraser $\mathrm{Cl}$, Hewitt GM. Founder takes all: density-dependent processes structure biodiversity. Trends Ecol Evol. 2013;28:78-85.

88. Moritz C, Fujita MK, Rosauer D, Agudo R, Bourke G, Doughty P, Scott M. Multilocus phylogeography reveals nested endemism in a gecko across the monsoonal tropics of Australia. Mol Ecol. 2015;25(6):1354-66.

89. Harris DJ, Carranza S, Arnold EN, Pinho C, Ferrand N. Complex biogeographical distribution of genetic variation within Podarcis wall lizards across the Strait of Gibraltar. J Biogeogr. 2002;29:257-1262.

90. Poulakakis N, Lymberakis P, Valakos E, Zouros E, Mylonas M. Phylogenetic relationships and biogeography of Podarcis species from the Balkan Peninsula, by Bayesian and maximum likelihood analyses of mitochondrial DNA sequences. Mol Phylogenet Evol. 2005;37:845-57.

91. McDevitt AD, Zub K, Kawałko A, Oliver K, Herman JS, Wójcik JM. Climate and refugial origin influence the mitochondrial lineage distribution of weasels (Mustela nivalis) in a phylogeographic suture zone. Biol J Linnean Soc. 2012;106:57-69.

92. While GM, Michaelides S, Heathcote RJ, MacGregor HE, Zajac N, Beninde J، Horváthová T. Sexual selection drives asymmetric introgression in wall lizards. Ecol Lett. 2015;18:1366-75.

93. Sosdian S, Rosenthal Y. Deep-sea temperature and ice volume changes across the Pliocene-Pleistocene climate transitions. Science. 2009;325:306-10.

94. Berger $\mathrm{WH}$, Jansen $\mathrm{E}$. Mid-pleistocene climate shift-the Nansen connection. The polar oceans and their role in shaping the global environment. 1994. p. 295-311

95. Hughes PD, Woodward JC, Gibbard PL. Middle Pleistocene cold stage climates in the Mediterranean: new evidence from the glacial record. Earth Planet Sci Lett. 2007;253:50-6.

96. Trincardi F, Cattaneo A, Asioli A, Correggiari A, Langone L. Stratigraphy of the late-Quaternary deposits in the central Adriatic basin and the record of shortterm climatic events. Memorie-Istituto Italiano di Idrobiologia. 1996;55:39-70.

97. Dumolin-Lapègue S, Demesure B, Fineschi S, Le Corre V, Petit RJ. Phylogeographic structure of white oaks throughout the European continent. Genetics. 1997;146:1475-88.

98. Marko PB, Hoffman JM, Emme SA, McGovern TM, Keever CC, Nicole CL. The 'Expansion-Contraction' model of Pleistocene biogeography: rocky shores suffer a sea change? Mol Ecol. 2010;19:146-69.

99. Říčanová Š, Koshev Y, Říčan O, Ćosić N, Ćirović D, Sedláček F, Bryja J. Multilocus phylogeography of the European ground squirrel: cryptic interglacial refugia of continental climate in Europe. Mol Ecol. 2013;22:4256-69.

100. Ho SYW, et al. Bayesian estimation of substitution rates from ancient DNA sequences with low information content. Syst Biol. 2011;60(3):366-75.

101. Crandall ED, Sbrocco EJ, DeBoer TS, Barber PH, Carpenter KE. Expansion dating: calibrating molecular clocks in marine species from expansions onto the Sunda Shelf following the Last Glacial Maximum. Mol Biol Evol. 2012;29:707-19.

102. Hewitt GM. Postglacial re-colonization of European biota. Biol J Linnean Soc. 1999;68:87-112.

103. Buades JM, Rodríguez V, Terrasa B, Perez-Mellado V, Brown RP, Castro JA, Ramon MM. Variability of the mc1r Gene in Melanic and Non-Melanic Podarcis lilfordi and Podarcis pityusensis from the Balearic Archipelago. PLoS One. 2013;8:e53088.

104. Sequeira F, Ferrand N, Harris DJ. Assessing the phylogenetic signal of the nuclear $\beta$-Fibrinogen intron 7 in salamandrids (Amphibia: Salamandridae). Amphibia-Reptilia. 2006;27(3):409-18.

105. Prychitko TM, Moore WS. The utility of DNA sequences of an intron from the $\beta$-fibrinogen gene in phylogenetic analysis of woodpeckers (Aves: Picidae). Mol Phylogenet Evol. 1997;8(2):193-204. 